\title{
Original
}

\section{Efecto de la terapia antirretroviral en la rehospitalización por enfermedad oportunista en Costa Rica}

\author{
Roy Wong-McClure', María-Paz León-Bratti ${ }^{2}$
}

1. Programa de Análisis y Vigilancia Epidemiológica

Caja Costarricense de Seguro Social

2. División de Inmunología, Servicio de Medicina Interna, Hospital México Caja Costarricense de Seguro Social y Escuela de Medicina, Universidad de Costa Rica.

Abreviaturas: CCSS, Caja Costarricense de Seguro Social; OMS-OPS,

Organización Mundial de la Salud Organización

Panamericana de la Salud; TARV,Terapia antirretroviral.

Correspondencia:

Dirección Postal. Apdo. 3791260 San José, Costa Rica Dirección Física: División de Inmunología, Laboratorio de Inmunología. Hospital México,

La Uruca. San José (Apdo 3791260)Correo electrónico: rwong@ccss.sa.cr $-$

\section{Resumen}

Objetivo: Caracterizar los egresos hospitalarios por VIH/Sida en el Hospital México, y evaluar la repercusión que la terapia antirretroviral (TARV) ha tenido en la probabilidad de reingreso por enfermedades oportunistas.

Metodología: Se recopilaron los datos de todos los pacientes egresados del Hospital México con diagnóstico de VIH/Sida, entre enero de 1995 y abril de 2003. El análisis descriptivo de la información se realizó utilizando el software SPSS, versión 12.0, con el cálculo de las medidas de tendencia central y dispersión para las variables cuantitativas y de las frecuencias para las variables cualitativas. Se calculó el análisis de sobrevida por medio del software Minitab, versión 12.2, con la definición de un nivel de significancia estadística menor o igual a 0.05. Como criterio de inclusión se seleccionaron todos los pacientes que egresados vivos de su primer internamiento con el diagnóstico de VIH/Sida y se dividieron en dos grupos según el año de su ingreso y su exposición a TARV. El primer grupo corresponde al período de enero de 1995 a diciembre de 1997, sin exposición a TARV, ya que los individuos del primer período que iniciaron TARV fueron excluidos del análisis. El segundo período involucra los egresos hospitalarios con diagnóstico de VIH/Sida entre 1998 y abril de 2003.
\end{abstract}

Resultados: Se analizaron los datos de 1081 egresos de 623 pacientes, un $85.7 \%$ de hombres, con una edad media de 36.8 años; y un 69.8\% de elos, solteros. El promedio de hospitalizaciones por año fue de 71 pacientes. La mortalidad general observada fue del $30 \%$, pero se observó una tendencia a disminuir. Solo un $42.5 \%$ de los egresos correspondían a asegurados directos. El análisis de sobrevida Kaplan Meier reveló que la media para desarrollar una segunda enfermedad oportunista fue de 466.2 días (IC95\% 382.8-549.7) para el grupo sin TARV, y de 1050. 5 días (IC95\% 952.1-1148.9) para el grupo con TARV, con diferencias significativas entre ambos períodos (Log Rank, $\mathrm{p}<0.05$ ). Este resultado no fue influenciado ni por el nivel de CD4, basal ni por la carga viral basal.

Conclusión: El número de egresos hospitalarios por VIH/Sida sigue aumentando. Se trata de una población joven, en su mayoría hombres solteros. La mortalidad intrahospitalaria es alta, aunque ha tendido a disminuir. El análisis de sobrevida comprueba la eficacia de la terapia antirretroviral para disminuir la ocurrencia de nuevas infecciones oportunistas y reinternamientos.

Descriptores: Terapia antirretroviral, VIH/Sida, enfermedades oportunistas

Key words: Antiretroviral therapy, HIV, AIDS, oportunistic infections.

Recibido: 14 de febrero de 2006

Aceptado: 8 de agosto de 2006 
La infección por el virus de inmunodeficiencia humana (VIH) que causa el Sida como parte de su evolución natural, se ha convertido en la pandemia, el evento médico y de salud pública más importante de nuestros tiempos, y destaca entre las principales enfermedades infecciosas en la historia de la humanidad ${ }^{1}$.

Su expansión ha sido universal, en crisis epidémicas sucesivas en diferentes regiones. La magnitud de la catástrofe en salud pública aún no ha sido valorada por algunos países. Sin embargo, se estima que en 2004 se infectaron con el VIH 4.9 millones de personas, y fallecieron por causas asociadas al VIH ${ }^{2}, 3.1$ millones.

Para diciembre de 2004 la Organización Mundial de la Salud (OMS) estimó que entre 35.9 y 44.3 millones de personas en el mundo vivían con el VIH; de estos individuos, entre 2 y 2.6 millones eran menores de 15 años; se reporta una tendencia creciente en el número de mujeres afectadas ${ }^{2}$.

América Latina ha presentado un incremento en el número de casos de nuevas infecciones por VIH, pasando de 190000 en 2002 a 240000 en 2004. Para diciembre de 2004 la OMS estimó que en la región vivían entre 1.3 y 2.2 millones de personas infectadas por el VIH, para una prevalencia del 0.5 al $0.8 \%{ }^{2}$.

En Costa Rica se reporta un acumulado de 2546 casos de Sida desde 1983 hasta diciembre de 2004, con un total de 1613 fallecidos y una incidencia anual, por millón de habitantes, de 17.01 para 2003, y una razón hombre - mujer para el mismo año, de $3.73^{3}$.

Clínicamente, la enfermedad por VIH se caracteriza por una inmunosupresión profunda y progresiva que, de no intervenirse conduce al individuo a la muerte por enfermedades oportunistas, infecciones o tumores ${ }^{4,5}$. Los gastos de atención médica de las enfermedades oportunistas son elevados y suelen involucrar hospitalizaciones prolongadas, incapacidades y pensiones prematuras. Dado que a nivel mundial el principal grupo afectado por la infección por VIH es el comprendido entre los 25 y los 40 años, los costos médicos y sociales para las economías han sido muy altos y han tenido repercusiones importantes, en los países en desarrollo.

Luego de la identificación del VIH como agente causal del Sida ${ }^{6}$, uno de los principales avances científicos ha sido el desarrollo de medicamentos antirretrovirales para tratar a los infectados ${ }^{7,8}$. La introducción de la TARV combinada ha cambiado la historia natural de la enfermedad, convirtiéndola de una de tipo mortal, en la mayoría de los casos, a una crónica, dado que los nuevos esquemas terapéuticos lograron una supresión viral completa por largos períodos ${ }^{9-11}$.

Múltiples estudios en países desarrollados han demostrado beneficios sustanciales con la TARV, en términos tanto de sobrevida como de calidad de vida de los pacientes con VIH/Sida ${ }^{9,10}$. Recientemente, investigaciones en países en desarrollo, como Brasil, han evidenciado beneficios similares ${ }^{12}$; también en Jamaica y Bermudas se ha reportado una reducción en la mortalidad y en las hospitalizaciones por infecciones oportunistas ${ }^{13}$.

Se ha comprobado la efectividad de la TARV tanto en prevenir enfermedades oportunistas como en mejorar la sobrevida en personas infectadas con VIH ${ }^{14-17}$.

Ensayos clínicos controlados en relación con la TARV, el riesgo de Sida o muerte en pacientes infectados es de la mitad en comparación con tratamientos menos potentes ${ }^{17}$.

Estudios observacionales han dado cuenta de que la TARV ejerce un efecto protector al momento de aparecer la primera enfermedad que define la condición de Sida, disminuyendo a cerca de un tercio el riesgo al compararse con la administración de monoterapia ${ }^{18-20}$.

Además, la TARV reduce la agresividad ante la presentación de patologías como el sarcoma de Kaposi ${ }^{21}$. A pesar de estos grandes beneficios y de las recomendaciones brindadas por la OMS-OPS ${ }^{13,22}$, el acceso a la terapia a nivel mundial ha sido muy limitado, probablemente por su alto costo ${ }^{6}$, dado que, por ejemplo, el costo anual directo para la atención de VIH en EEUU excede los US \$20 000 por individuo ${ }^{23}$. En los países de bajos y medianos ingresos, solo alrededor de 440000 personas recibían TARV para junio de 2004, lo que significa que 9 de cada 10 de quienes requieren el tratamiento, no lo estaban recibiendo; de continuar con estas bajas coberturas, en los próximos dos años morirán entre cinco y seis millones de personas por consecuencia del Sida ${ }^{2}$. América Latina y el Caribe disponen de una cobertura estimada del 55\% de la población adulta afectada con el VIH ${ }^{2}$.

La TARV se introdujo en la Caja Costarricense de Seguro Social (CCSS) en octubre de 1997. Sin embargo, no se dispone de un estimado acerca del costo de la atención integral por paciente.

Dado que en el país la eficiencia de la TARV no ha sido objetivamente evaluada hasta el momento dentro de la seguridad social, el presente trabajo evalúa la repercusión que esta ha tenido en la rehospitalización por enfermedades oportunistas en el Hospital México, en San José, Costa Rica.

\section{Materiales y métodos}

La investigación corresponde a un estudio de cohorte retrospectivo, que define con variable de exposición el empleo de la TARV, y como evento, la rehospitalización por enfermedad oportunista. El período de observación establecido fue entre enero de 1995 y abril de 2003. 


\section{Manejo de bases de datos}

Se obtuvo la información del registro de egreso hospitalario del Hospital México con el diagnóstico de VIH/sida, según la clasificación internacional de enfermedades (CIE), en sus novena y décima revisiones, entre enero de 1995 y abril de 2003.

Para los análisis de la probabilidad de reingreso por enfermedad oportunista se depuró la información excluyendo a quienes fallecieron en la primera hospitalización. Estos casos se estratificaron en dos grupos, según la fecha de egreso hospitalario de la primera vez. El primer período correspondió a los pacientes egresados entre enero de 1995 y diciembre de 1997, es decir, al grupo de pacientes no expuestos a TARV. El segundo período comprendió los egresados entre enero de 1998 y abril de 2003, y representa a los pacientes que recibieron TARV. Los que formaron parte del primer grupo fueron excluidos del segundo y se concluyó su período de observación el 31 de diciembre de 1997.

Se complementó la información correlacionando la base de datos del egreso con la de la Clínica de VIH del mismo centro hospitalario, para tomar en consideración el valor basal de linfocitos T CD4+ y carga viral.

El grupo expuesto a TARV se estratificó para posterior análisis, según el conteo de linfocitos T CD4+, en dos grupos: los que tenían un CD4 menor de 200 células $/ \mathrm{mm}^{3}$ y los que tenían un CD4 por encima de tal valor.

El grupo de pacientes expuestos a TARV también se estratificó, según la carga viral, en dos subgrupos: los que tenían una carga viral superior o igual a 75000 y los que tenían una carga viral inferior a esta.

\section{Análisis estadístico}

El análisis descriptivo de la información de ambos grupos se realizó por medio del software SPSS, versión 12.0, con el cual se calcularon las frecuencias de las variables cualitativas y de las medidas de tendencia central y dispersión de las variables cuantitativas.

Se llevó a cabo una prueba de homogeneidad de Chi-Cuadrado $\left(\chi^{2}\right)$ entre ambos grupos, para las variables categóricas y pruebas de t-Student, con el fin de comparar las medias de las variables cuantitativas y evaluar la homogeneidad entre grupos al inicio del período de observación.

Se realizó un análisis de sobrevida no paramétrico de Kaplan-Meier, utilizando el software Minitab ${ }^{14,15}$, versión 12.2, con la aplicación de la prueba de Log Rank para la comparación entre grupos, con la definición de un nivel de significancia menor a $0.05(\alpha<0.05)$. El análisis de sobrevida fue realizado posteriormente con la estratificación de los pacientes con TARV (grupo expuesto), según el nivel basal de linfocitos T CD4+ y carga viral.

\section{Resultados}

Entre enero de 1995 y abril de 2003 se registraron un total de 1080 egresos hospitalarios con el diagnóstico de VIH/sida del Hospital México, los cuales correspondieron a un total de 623 pacientes, para 1.6 internamientos por paciente, en el período en estudio.

Un $85.7 \%$ de los pacientes pertenecían al sexo masculino; en cuanto al estado civil, un $70.0 \%$ eran solteros, un $16.5 \%$ casados y un $13.5 \%$ tenían otras condiciones. La media de edad a la fecha del egreso fue de 36.8 años. El promedio de hospitalizaciones por año fue de 115 pacientes (rango 56-152).

El número de egresos por año aumentó en forma progresiva de 1994 -56 egresos- hasta 1998 -152 pacientes con este diagnóstico-. Entre 1999 y 2001 los egresos por esta causa mostraron una tendencia a disminuir para elevarse luego en 2002. En relación con los días de estancia hospitalaria, el promedio varió de 11.4 días (IC95\% 10.8-13.9), como valor más bajo para 1997 a 12.1 días (IC95\% 13.718.6), como el más elevado para 1999.

Del total de individuos egresados por VIH/sida, 190 (30\%) fallecieron durante el primer internamiento. La edad media de estos pacientes al momento del deceso fue de 39.5 años (IC95\% 37.8-41.1) y el promedio de días estancia para los fallecidos fue de 14.8 (IC95\% 12.6-17.0), con una tendencia decreciente durante el periodo de observación (Figura 1).

De total de pacientes seleccionados y que no fallecieron durante la primera hospitalización, 214 (45.0\%) reingresaron por presentar alguna enfermedad oportunista durante el período de estudio para ambos grupos.

La comparación de las variables categóricas y cuantitativas al inicio del período de observación no evidenció

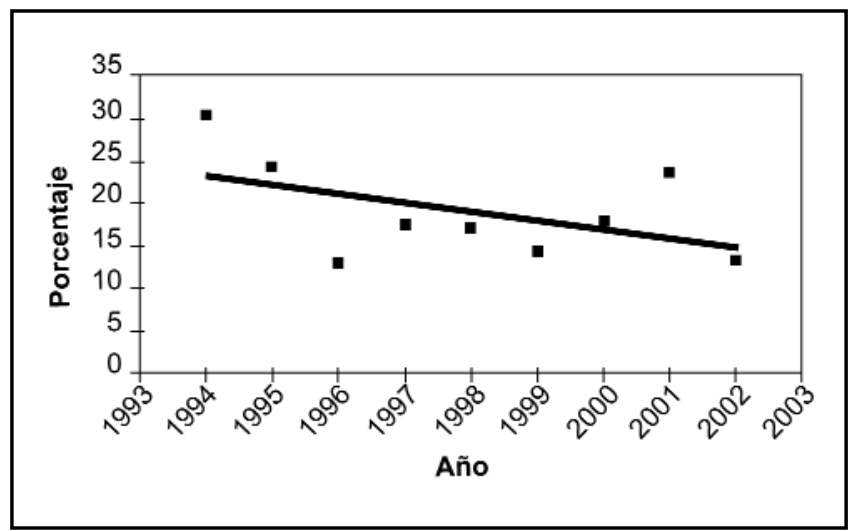

Figura 1. Mortalidad intrahospitalaria por VIH/Sida, según año. Hospital México. 1994-2002 


\begin{tabular}{|c|c|c|c|c|c|c|c|}
\hline \multirow[t]{2}{*}{ Variable } & \multicolumn{3}{|c|}{ Grupo sin TARV } & \multicolumn{3}{|c|}{ Grupo con TARV } & \multirow{2}{*}{$\begin{array}{c}\mathrm{t} \text {-student } \\
\mathrm{P}\end{array}$} \\
\hline & $n$ & Promedio & IC95\% & $\mathrm{n}$ & Promedio & IC95\% & \\
\hline Edad (años) & 136 & 34,8 & $33.1-36.6$ & 196 & 35.9 & $34.5-37.4$ & 0.85 \\
\hline $\begin{array}{l}\text { CD4+ basal } \\
\text { (cels/MMS) }\end{array}$ & 27 & 258.1 & $167.2-348.9$ & 123 & 298.1 & $250.6-345.1$ & 0.67 \\
\hline $\begin{array}{l}\text { CV basal } \\
\text { (copias/) }\end{array}$ & 24 & 103530 & $3.3-171.8 \times 103$ & 101 & 94136 & $6.77-12.95 \times 104$ & 0.55 \\
\hline Días de estancia & 136 & 14.7 & $12.4-17.0$ & 196 & 15.8 & $14.0-17.6$ & 0.31 \\
\hline
\end{tabular}

diferencias significativas en ninguna de las características basales de los grupos (Cuadro 1).

Al realizar el análisis de sobrevida de Kaplan Meier se determinó que la media para desarrollar una segunda enfermedad oportunista fue de 466.2 días (IC95\% 382.8-549.7) para el grupo no expuesto a TARV, y de 1050.4 días (IC95\% 952.0-1148.9) para el expuesto, lo que demuestra diferencias estadísticamente significativas entre ambos períodos (Log-Rank 20.19, $\mathrm{p}<0.001)$.

En el grupo no expuesto, la mitad (68/136) de la población había reingresado por enfermedad oportunista a los 254 días de seguimiento desde su primera hospitalización. En el expuesto, durante el mismo tiempo de seguimiento solo el 35\% (68/197) de los individuos $(\mathrm{p}<0.001)$ había presentado un reingreso por enfermedad oportunista (Figura 2).

Al estratificar el grupo expuesto a TARV, según los niveles basales de linfocitos de T CD4+., no se obtuvieron diferencias significativas en el análisis de reingreso por

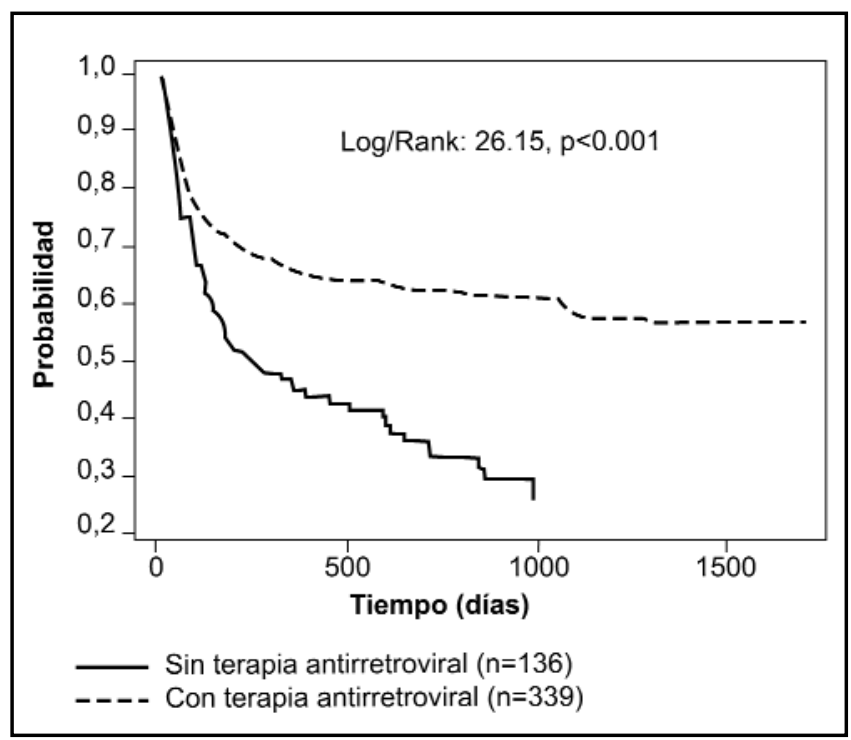

Figura 2. Estimación por método de Kaplan-Meier de tiempo de aparición de enfermedad oportunista en pacientes VIH/sida, según período, sin y con TARV. Hospital México. Enero 1995-abril 2003. enfermedad oportunista (Log-Rank=0.53, p=0.75) (Figura 3). Similar resultado se obtuvo con la estratificación por carga viral basal (Log-Rank=0.11, $\mathrm{p}=0.73$ ) (Figura 4).

\section{Discusión}

El estudio realizado analizó el comportamiento de la hospitalización de los pacientes con VIH/sida en una muestra que representa la quinta parte de la población afectada, según las estadísticas del Ministerio de Salud de Costa Rica.

En relación con la estancia promedio de hospitalización de los pacientes estudiados, se evidenció poca variabilidad entre los años de observación, y se determinó muy cercana al promedio de estancia hospitalaria por otras causas en los mismos años en el Departamento de Medicina Interna (promedio de 12 días).

El análisis de la mortalidad para los pacientes con VIH/sida hospitalizados, mostró una clara tendencia al descenso, aspecto en el que es de importancia recalcar que la gran mayoría de los pacientes fallecen durante su primera hospitalización, antes del inicio de la TARV o habiéndola recibido por un corto período, lo que concuerda con lo descrito por Hammer y colaboradores.

El análisis de sobrevida de Kaplan-Meier, donde se evaluó la rehospitalización por enfermedad oportunista, evidenció que los pacientes con TARV duplicaron el tiempo libre de una enfermedad oportunista en relación con el grupo no expuesto, resultado que no se vio influenciado por los niveles basales de CD 4+ ni carga viral, hallazgo también descrito en diversas investigaciones que demostraron la reducción en la probabilidad de la condición de sida y de muerte en pacientes con infección demostrada.

En diversas investigaciones los niveles basales de CD4+ y los de carga viral han demostrado influir en la morbimortalidad de la enfermedad, situación que en este estudio no se evidenció, posiblemente por la falta de potencia estadística, por efecto del tamaño de la muestra. 


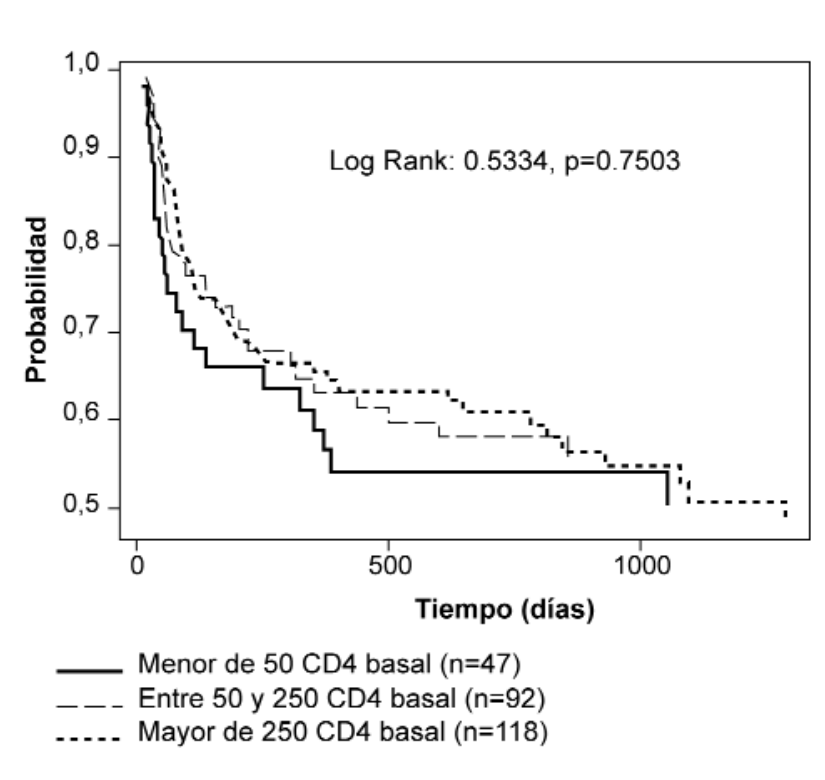

Figura 3. Determinación por método de Kaplan-Meier de tiempo de aparición de enfermedad oportunista, según conteo basal de linfocitos T CD4+. Hospital México. Enero 1998-abril 2003.

El sistema de seguridad social costarricense ha logrado grandes beneficios en la salud y el bienestar de la población; con una inversión económica relativamente pequeña, debido al bajo producto interno bruto del país, se han logrado índices de salud similares a los de países desarrollados y se cuenta con gran diversidad y accesibilidad de los servicios.

Sin embargo, los avances científicos en el manejo y tratamiento de las enfermedades establecen presión sobre el sistema, al crear la necesidad de brindar terapias muy caras a pequeñas fracciones de la población con enfermedades relativamente poco frecuentes.

Los resultados brindan, por tanto, clara evidencia de dos logros obtenidos con la implementación de la TARV en Costa Rica: un descenso en la mortalidad hospitalaria por VIH/Sida y un significativo aumento del período libre de enfermedad oportunista en los pacientes que la reciben.

\section{Abstract}

Aim: To characterize the hospitable debits by VIH/AIDS in the Hospital Mexico, and to evaluate the repercussion that the antiretroviral therapy (ARVT) has had in the probability of re-hospitallization by opportunistic diseases in these individuals.

Methodology: the data of all the withdrawn patients of the Hospital Mexico were compiled with HIV/AIDS diagnosis between January of 1995 and April of the 2003. The

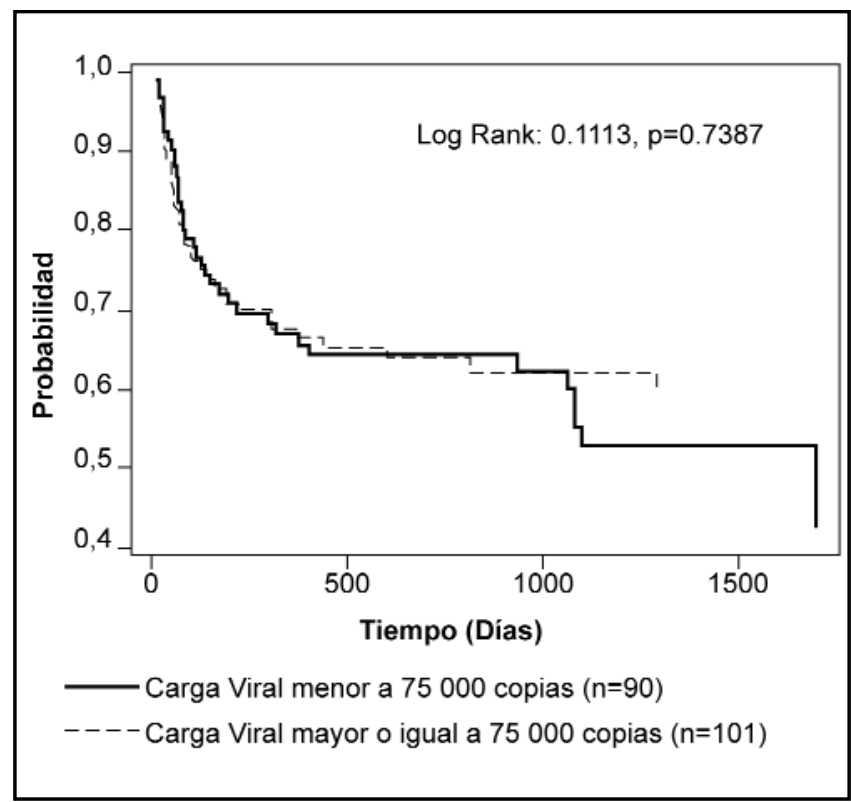

Figura 4. estimación por método de Kaplan-Meier de tiempo de aparición de enfermedad oportunista, según nivel basal de carga viral. Hospital México. Enero 1998-abril 2003.

descriptive analysis of the information was made using software SPSS version 12.0 with the calculation of the measures of central tendency and dispersion for the quantitative variables and calculation of the frequencies for the qualitative variables.

The calculation of the survival analysis was made by the software Minitab vesión 12,2 with the definition of a statistical significance level of smaller or equal to 0.05 . As inclusion criterion all the patients selected themselves who withdrew alive of their first hospitalization with the HIV/AIDS diagnosis and divided in two groups according to the year of their entrance and its exhibition to ARVT. The first group corresponds to the period of January of 1995 to December de1997 and not exposed to ARVT, the individuals of the first period that initiated ARVT were excluded from the analysis. The second period of 1998 to April of 2003 involves the hospitable debits with HIV/ AIDS diagnosis.

Results: The data of 1081 debits corresponding to 623 patients, 85,7\% men were analyzed, with an average age of 36,8 years, $69,8 \%$ were unmarried. The average of hospitalizations per year was of 70,9 patients. Observed general mortality was of $30 \%$, but it was observed in same a tendency to diminish. Only $42,5 \%$ of all the debits corresponded to direct insured.

The Kaplan Meier analysis revealed that the average to develop a second opportunistic disease was of 466,2 days (IC95\% 382.8-549.7) for the group without ARVT and of 1050,5 days (IC95\% 952.1-1148.9) for the group under the therapy with significant differences between both periods (Log Rank, $\mathrm{p}<0.05)$. This result was influenced neither by the basal level of CD4 nor by the basal viral load. 
Conclusion: The number of hospitable debits by VIH/AIDS continues increasing. One is a young population, in his majority men, unmarried. Intrahospitable mortality is high, although it has tended to diminish. The survival analysis verifies the effectiveness in our means of the antiretroviral therapy to diminish the occurrence of new opportunistic infections and new hospitalizations.

\section{Referencias}

Fauci AS. The AIDS epidemic — consideration for the 21st century. N Engl J Med. 1999; 341:1046-1050.

2. Organización Mundial de la Salud-ONUSIDA. Situación de la epidemia de SIDA: 2004. Diciembre 2004.

3. Unidad VIH/SIDA/ITS de la Organización Panamericana de la Salud. Vigilancia del Sida en las Américas. Washington; junio 2005. pp.31

4. Kaposi's sarcoma and pneumocystis pneumonia among homosexual men—New York city and California. Morb Mortal Wkly Rep 1981; 30:305-308.

5. Vergis EN, Mellors JW. Natural history of HIV-1 infection. Infect Dis Clin North Am 2000; 14:809-825.

6. Barré-Sinoussi $\mathrm{F}$ et al. Isolation of a T-lymphotropic retrovirus from a patient at risk for acquired simmune deficiency syndrome (AIDS). Science 1983; 220:868-871.

7. Fauci AS. HIV and AIDS: 20 years of science. Nat Med 2003; 9:839843.

8. Ponerantz RJ, Horn DL. Twenty years of therapy for HIV-1 infection. Nat Med 2003; 867-873.

9. Detels R, Muñoz A, MacFarlane G, Kingsley LA, Margolick JB, Giorgi J, et al. Effectiveness of potent antiretroviral therapy on time to AIDS and death in men with known HIV infection duration. JAMA 1998; 280:1497-1503.

10. Kholoud P and CASCADE Collabortion. Survival after introduction of HAART in people with known duration of HIV-1 infection. Lancet 2000; 355:1158-1159.

11. Panel on clinical practices for treatment of HIV infection, Department of Health and Human Services. Guidelines for the use of antiretroviral agents in HIV-1-infected adults and adolescents. October 6, 2005. pp. 118
12. Marins JR, Jamal LF, Chen SY, Barros MB, Hudes ES, Barbosa AA, Chequer P, Teixeira PR, Hearts N. Dramatic improvement in survival among adult Brazilian AIDS patients. AIDS 2003; 17:1675-1682.

13. OPS/OMS. Vigilancia del Sida en las Américas, Informe Bianual. Junio 2002.

14. Cain LE, Cole SR, Chmiel JS, Margolick JB, Rinaldo CR, Detels R. Effect of highly active antiretroviral therapy on multiple aids-defining illnesses among male HIV seroconverters. Am J Epidemiol 2006;163:310-315

15. Cameron DW, Heath-Chiozzi M, Danner S, et al. Randomised placebo-controlled trial of ritonavir in advanced HIV-1 disease. The Advanced HIV Disease Ritonavir Study Group. Lancet 1998;351:543-9.

16. Gulick RM, Mellors JW, Havlir D, et al. Treatment with indinavir, zidovudine, and lamivudine in adults with human immunodeficiency virus infection and prior antiretroviral therapy. $\mathrm{N}$ Engl $\mathrm{J}$ Med 1997;337:734-9.

17. Hammer SM, Squires KE, Hughes MD, et al. A controlled trial of two nucleoside analogues plus indinavir in persons with human immunodeficiency virus infection and CD4 cell counts of 200 per cubic millimeter or less. AIDS Clinical Trials Group 320 Study Team. N Engl J Med 1997;337:725-33.

18. Egger M, Hirschel B, Francioli P, et al. Impact of new antiretroviral combination therapies in HIV infected patients in Switzerland: prospective multicentre study. BMJ 1997;315:1194-9.

19. Mocroft A, Katlama C, Johnson AM, et al. AIDS across Europe, 1994-98: the EuroSIDA study. Lancet 2000;356:291-6.

20. Detels R, Munoz A, McFarlane G, et al. Effectiveness of potent antiretroviral therapy on time to AIDS and death in men with known HIV infection duration. Multicenter AIDS Cohort Study Investigators. JAMA 1998;280:1497-503.

21. Nasti G, Martellotta F, Berretta M, Mena M, Fasan M, Di Perri G et al. Impact of highly active antiretroviral therapy on the presenting features and outcome of patients with acquired mmunodeficiency syndrome-related Kaposi sarcoma. Cancer 2003;98(11):2440-2446.

22. Organización Panamericana de la Salud-Organización Mundial de la Salud. 128a Sesión del Comité Ejecutivo. Junio, 2001.

23. Hellinger FJ, Fleishman JA. Estimating the national cost of treating people with HIV disease: patient, payer, and provider data. J Acquir Immune Defic Syndr 2000; 24:182-188. 